\title{
Editorial Appreciation
}

\author{
John Leibacher ${ }^{1,2,3}(\mathbb{D}) \cdot$ Cristina H. Mandrini ${ }^{4}(\mathbb{D}$ \\ Lidia van Driel-Gesztelyi ${ }^{5,6,7}$ (D) \\ Michael S. Wheatland ${ }^{8}$
}

Published online: 15 January 2018

(C) Springer Science+Business Media B.V., part of Springer Nature 2018

We are pleased to acknowledge, with sincere thanks, the following colleagues who supported the community by reviewing articles for Solar Physics during 2017:

\section{Referee of three articles:}

Edward Cliver.

Referees of two articles:

Paul Cally, Robert Cameron, Kazumasa Iwai, Yang Liu, Arturo López Ariste, Jean-Pierre Raulin, Kiyoto Shibasaki, Ilya Usoskin, and Brian Wood.

\footnotetext{
J. Leibacher

john.leibacher.sola@gmail.com

C.H. Mandrini

mandrini@iafe.uba.ar

L. van Driel-Gesztelyi

lidia.vandriel@obspm.fr

M.S. Wheatland

michael.wheatland@sydney.edu.au

1 National Solar Observatory, Tucson, AZ, USA

2 Institut d'Astrophysique Spatiale - UMR-8617, Université Paris-Sud/CNRS, Orsay, France

3 Lunar and Planetary Laboratory, University of Arizona, Tucson, AZ, USA

4 Instituto de Astronomía y Física del Espacio, IAFE, Consejo Nacional de Investigaciones

Científicas y Técnicas - Universidad de Buenos Aires, Buenos Aires, Argentina

5 Observatoire de Paris, LESIA, CNRS, UPMC Université Paris-Diderot, Meudon, France

6 Mullard Space Science Laboratory, University College London, Dorking, UK

7 Konkoly Observatory, Hungarian Academy of Sciences, Budapest, Hungary

8 Sydney Institute for Astronomy, The University of Sydney, NSW, Sydney, Australia
} 


\section{Referees of one article:}

Valentina Abramenko, Loren Acton, Costas Alissandrakis, Tanja Amerstorfer, Sergey Anfinogentov, Charles Arge, Rainer Arlt, Inigo Arregui, Markus Aschwanden, Andrés Asensio Ramos, Frédéric Auchère, Taeil Bai, Horst Balthasar, Dipankar Banerjee, Supratik Banerjee, Anatoly Belov, Arnold Benz, Daniel Berdichevsky, Thomas Berkefeld, Pietro Bernasconi, Luca Bertello, Irina Bilenko, Mario Bisi, Monica Bobra, Joseph Borovsky, Volker Bothmer, Axel Brandenburg, Anne-Marie Broomhall, Rolf Bütikofer, Ana Cadavid, Iver Cairns, Hilary Cane, Roberto Casini, Gianna Cauzzi, Jongchul Chae, Paul Charbonneau, Bin Chen, Christopher Chen, Xin Cheng, Mark Cheung, Kyung-Suk Cho, Steven Christe, Frédéric Clette, Manuel Collados Vera, Hebe Cremades, Serena Criscuoli, Norma Crosby, André Csillaghy, Maher Dayeh, Craig DeForest, Veronique Delouille, José Carlos del Toro Iniesta, Pascal Démoulin, Linhua Deng, Carsten Denker, Leonid Didkovsky, Mark Dierckxsens, Mausumi Dikpati, Turlough Downes, Thierry Dudok de Wit, Mateja Dumbović, Frederic Effenberger, Eugene Engelbrecht, Géza Erdős, Stefan Eriksson, Paul Evenson, Charles Farrugia, William Feldman, Shiwei Feng, Song Feng, Gregory Fleishman, Lyndsay Fletcher, Andrzej Fludra, Peter Foukal, Manolis Georgoulis, Guillermo Giménez de Castro, Simon Good, Marcel Goossens, Nat Gopalswamy, Lucie Green, Margit Haberreiter, Gerhard Haerendel, Rajkumar Hajra, John Hardy, Petr Heinzel, Carl Henney, Phillip Hess, Andrew Hillier, K.M. Hiremath, J. Todd Hoeksema, Timothy Howard, Qiang $\mathrm{Hu}$, Zhenghua Huang, Neal Hurlburt, Andrew Inglis, Jack Ireland, Bernard Jackson, Kiran Jain, Miho Janvier, Javaraiah Javaraiah, Elizabeth Jensen, Jie Jiang, Andrew Jordan, Primož Kajdič, Kazutaka Kaneda, Marian Karlický, Christina Kay, Maria Kazachenko, Ali Kilcik, Emilia Kilpua, Rok-Soon Kim, Michael Kirk, Leonid Kitchatinov, Irina Kitiashvili, Karl-Ludwig Klein, Zoltán Kolláth, Rudi Komm, Eduard Kontar, Sylvain Korzennik, József Kóta, Július Koza, Kamen Kozarev, Masahito Kubo, Therese Kucera, K. Kudela, Pankaj Kumar, Elena Kupriyanova, Ryun Young Kwon, Andreas Lagg, Jan Langfellner, Marian Lazar, Laure Lefèvre, K.D. Leka, Susan Lepri, Bo Li, Haosheng Lin, Charles Lindsey, Roberto Lionello, Rui Liu, Ying Liu, Ramon Lopez, Jeffrey Love, Christopher Lowder, Noé Lugaz, Zuleika Luz Sodré, Jasmina Magdalenic, Shane Maloney, Sudip Mandal, Katsuhide Marubashi, Helen Mavromichalaki, Mona Mays, Scott McIntosh, Valentin Melnik, Travis Metcalfe, Grzegorz Michalek, Mark Miesch, Kris Murawski, K.N. Nagendra, Yury Nagovitsyn, Valery Nakariakov, Ralph Neuhäuser, Teresa Nieves-Chinchilla, Alexander Nindos, Naoto Nishizuka, Nariaki Nitta, Aimee Norton, Vladimir Obridko, Ramón Oliver, Evangelos Paouris, Susanna Parenti, Jay Pasachoff, Spiros Patsourakos, Tiago Pereira, Rui Pinto, Mykola Pishkalo, Stefaan Pommé, Werner Pötzi, Jiong Qiu, Ramesh Rajaram, Nour-Eddine Raouafi, Donald Reames, Stéphane Régnier, Michael Reiner, Ian Richardson, Bernard Roberts, Luciano Rodriguez, Alexis Rouillard, Luc Rouppe van der Voort, Michael Ruderman, Basilio Ruiz Cobo, Daniel Ryan, James Ryan, Antonia Savcheva, Thomas Schad, Philip Scherrer, Dirk Schmidt, Wolfgang Schmidt, Jesper Schou, Hannah Schunker, Michal Sobotka, P. Sriramachandran, Guillermo Stenborg, Alexander Stepanov, Prasad Subramanian, Davor Sudar, Xudong Sun, Leif Svalgaard, Baolin Tan, James Tappin, Manuela Temmer, Golla Thejappa, Shin Toriumi, Durgesh Tripathi, Sushanta Tripathy, Javier Trujillo Bueno, Bruce Tsurutani, Sylvaine Turck-Chièze, Roger Ulrich, Ignacio Ugarte Urra, Tom Van Doorsselaere, José Vaquero, Alberto Vásquez, Marco Velli, Panditi Vemareddy, Giuliana Verbanac, Meetu Verma, Luis Vieira, Angelos Vourlidas, Rui Wang, Alexander Warmuth, Harry Warren, Takashi Watanabe, David Webb, Brian Welsch, Stephen White, Peter Williams, Thomas Woods, Chin-Chun Wu, Anthony Yeates, Peter Young, Wenyuan Yu, Loukas Zachilas, Luca Zangrilli, Jie Zhang, Jun Zhang, Liang Zhao, Shuang Zhao, Nadezhda Zolotova, Yannis Zouganelis, and Pietro Zucca. 
We would like to thank Frédéric Auchère, Robert Cameron, Roberto Casini, Mark Cheung, Mateja Dumbović, Stefan Eriksson, Margit Haberreiter, Carl Henney, Qiang Hu, Kazumasa Iwai, Yang Liu, Katsuhide Marubashi, Nariaki Nitta, Basilio Ruiz Cobo, Phillip Scherrer, Manuela Temmer, Bruce Tsurutani, and Takashi Watanabe for particularly meritorious service in reviewing.

We wish to express our thanks and appreciation to the members of the Editorial Board, Editorial Board Chair Janet Luhmann, Memoir Committee Chair Edward Cliver, Guest Editors Xóchiti Blanco-Cano, Eduard Kontar, Alexander Nindos, Nandita Srivastava, Nariaki Nitta, and Jie Zhang, Associate Editors Rachel Howe and Matthew Owen, and our collaborators in the management, editing, and production of Solar Physics: Swathi Alagesan, Astrid Peter, Eric Pieren, Frank Schulz, Linas Stonys, and Vygintas Vilimas. 